\title{
Purification and Analysis of Synthetic Nucleic Acids and Components
}

\section{INTRODUCTION}

$\mathbf{P}$

urification and analysis are obviously essential to studies involving synthetic nucleic acids, but choice of the most appropriate technique, which ultimately depends on the final use for the oligonucleotide, is not always obvious. An overview unit (UNIT 10.3) discusses alternative techniques and provides information necessary for the reader to determine the most appropriate technique for a particular application of interest. For example, oligonucleotides to be used for sequencing or as PCR primers do not need to be as rigorously pure as those to be used for cloning or X-ray crystallography studies. The vast majority of oligonucleotides are purified by three techniques: polyacrylamide gel electrophoresis (PAGE; UNIT 10.4), high-performance liquid chromatography (HPLC; UNIT 10.5), or commercially available cartridges (UNIT 10.7). The overview unit contains basic information about synthesis, including evaluation of coupling yields, and options for deprotection, workup, purification, and quantification.

The most common technique for purification of oligonucleotides to be used as primers in PCR and sequencing is polyacrylamide gel electrophoresis. UNIT 10.4 provides detailed procedures for sample preparation, running gels, and visualization and isolation of oligonucleotides.

UNIT 10.5 on HPLC analysis and purification of synthetic nucleic acids includes basic information and procedures using both reversed-phase and anion-exchange HPLC. Cartridge techniques for oligonucleotide purification and desalting have become very popular because of their speed and efficiency. UNIT 10.7 provides the reader the background necessary to effectively use this technique.

Another method for separation of oligonucleotides is capillary electrophoresis (CE). Because of the small capillary diameters and high separating voltages used, CE is fast and offers high sensitivity and resolution (e.g., single-nucleotide resolution for nanogram quantities of fragments up to several hundred base pairs). UNIT 10.9 describes theory, instrumentation, and methods for analysis of oligonucleotides by CE.

The composition of synthetic oligonucleotides should always be confirmed. Recently, mass spectrometry has developed as the most efficient tool for rapid analysis of short oligonucleotides $(<40 \mathrm{nt})$. A molecular ion mass determination along with either PAGE or HPLC data is generally considered sufficient for the confirmation of oligonucleotide integrity and purity. MALDI-TOF mass analysis has become especially commonplace and is now routinely used by commercial oligonucleotide synthesis vendors.

Two different mass spectrometry techniques, matrix-assisted laser desorption/ionization time-of-flight (MALDI-TOF; UNIT 10.1) and electrospray ionization (ESI) mass spectrometry (UNIT 10.2) are particularly useful. The differences between the techniques, as well as the advantages and disadvantages of each, are compared. Both units contained detailed instruction for sample preparation and useful troubleshooting tips. Both techniques can be used to sequence oligonucleotides, and the fragment pattern in ESI mass spectrometry may be helpful in confirming the identity of oligonucleotide modifications. This kind of

Purification and

Analysis of Synthetic Nucleic Acids and Components

10.0.1

Supplement 13 
a more detailed analysis may be important for characterization of modified oligonucleotides for which conventional gel-based sequencing assays are not informative.

Base composition analysis, described in UNIT 10.6, is an important tool for establishing the presence and integrity of modified bases in synthetic oligonucleotides. Snake venom phosphodiesterase, a $3^{\prime}$ to $5^{\prime}$ exonuclease, is capable of degrading oligonucleotides containing modified bases. In conjunction with monomer standards, HPLC nucleoside analysis of enzyme-degraded oligonucleotides is a powerful tool for confirming the presence and quantifying modified nucleosides.

The oxidation of DNA is mediated by many different xenobiotics, including DNA-damaging drugs, substances present in the environment, and radiation. Determination of the pathway of oxidation is an important step in understanding the mechanism of DNA degradation by these substances. In UNIT 10.8, Awada and Dedon provide experimental methods for determining the site and, hence, mechanism of oxidation of DNA. These methods allow one to distinguish pathways that involve $1^{\prime}, 3^{\prime}, 4^{\prime}$, and $5^{\prime}$ hydrogen atom abstraction.

Donald E. Bergstrom 